\title{
Squamous cell carcinoma in a pre-existing adult mature Sacro-Coccygeal teratoma: A rare case with review of literature
}

\author{
Ranjan Agrawal ${ }^{1, *}$, Jagdamba Sharan ${ }^{2}$, Parbodh Kumar ${ }^{3}$ \\ ${ }^{1}$ Professor, Dept. of Pathology \& Surgery, Rohilkhand Medical College \& Hospital, Bareilly, Uttar Pradesh, India
}

*Corresponding Author:

Email: drranjan68@gmail.com

\begin{abstract}
We report a case of sacrococcygeal teratoma with squamous cell carcinomatous transformation in a 40-year-old lady. This is an infrequent location for teratoma in adults and malignant transformation has rarely been described. The uniqueness of the present case was the presence of congenital sacrococcygeal teratoma presenting in adulthood with malignant transformation.
\end{abstract}

Keywords: Malignant transformation, Sacrococcygeal, Squamous cell carcinoma, Teratoma.

Received: $04^{\text {th }}$ July, 2017

\section{Introduction}

Teratomas are found with decreasing frequency in the gonads (ovaries and testis), mediastinum, retroperitoneal space, sacrococcygeal region, pineal and other intracranial locations. ${ }^{1,2}$ Sacrococcygeal teratomas are congenital tumour that develop embryologically from multipotent cells and enlarge as pre and post sacral mass. As per their degree of maturation, they are classified as mature, immature, and malignant. We present an unusual case of congenital sacrococcygeal teratoma in a 40 year old female, later undergoing malignant transformation.

\section{Case Report}

A 40 year old female presented with an increasing swelling in the lower back since last 3 months. The swelling was present since birth, but had increased in size recently. There was no other significant history. Her menstrual history was normal with regular cycles of normal duration and flow. Patient was married, had 6 children all born by normal vaginal delivery at home. She recently developed mild fever and sudden significant increase in the size of the swelling 3 months back for which a local practitioner was consulted who aspirated copious amount of purulent fluid $(300-400 \mathrm{ml})$ from the swelling. After two-and half months the swelling recurred for which she was referred to this tertiary care hospital.

On local examination a swelling was noted in the sacral region measuring $12 \mathrm{~cm} \times 15 \mathrm{~cm}$ in size covered with dark coloured skin. The local temperature was not raised. The swelling was non-tender, fluctuation present, non-reducible or compressible with no abnormal pulsations. The skin over the swelling was tethered in the center but at the periphery it was free from the swelling. Another swelling was present just below the coccyx about $8 \mathrm{~cm} \times 6 \mathrm{~cm}$ in size and communicating with the swelling in the sacral region as on pressing either of the swelling the other swelling increased in size due to movement of fluid (cross fluctuation sign positive).

Routine blood tests were within normal limits. The pus which was drained out showed plenty of pus cells and culture isolated coagulase negative staphylococci sensitive to most antibiotics. No acid fast bacilli were present. USG abdomen did not show any significant abnormality except for incidental gall stones. MRI showed a large lobulated multiseptated heterogenously hyperintense altered signal intensity mass lesion at posterior aspect of sacrococygeal region, with no evidence of osseous destruction, intra- pelvic extension or inflammatory changes in the peripheral myofascial plane. Under epidural anaesthesia through a vertical slightly curved incision the tumour was explored. Skin flaps were raised and the tumour was dissected free from the skin and adjacent tissues. There were three extensions of the tumour, one in the sacral region, second in the coccygeal region and third towards the left lateral side away from the midline reaching up to the skin. All the three extensions were communicating with each other. Grossly, the tumour measured more than $25 \times 18 \mathrm{~cm}$ in size (Fig.1).

The tumour was adherent to the sacrum posteriorly at the site of the junction of the three extensions which was fulgurated, two drains 
placed and the wound closed. The histopathological examination of the resected specimen suggested mature teratoma with areas of squamous cell carcinoma changes i.e., Teratoma with malignant transformation (Figs. 2 and 3). The malignant squamous part showed positivity with Cytokeratin (Fig. 2b). Postoperative period was uneventful and the patient was discharged with an advice for regular follow up.

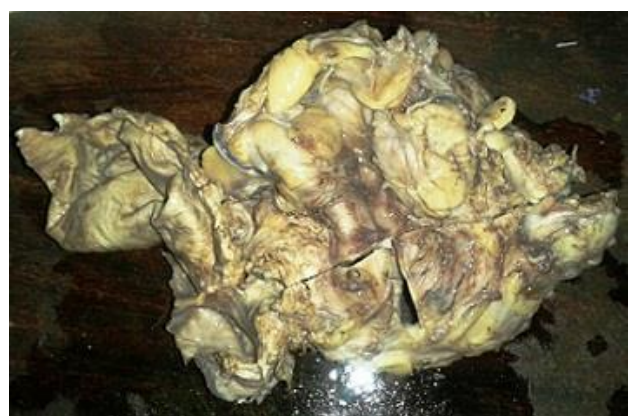

Fig.1: Gross appearance of the resected specimen

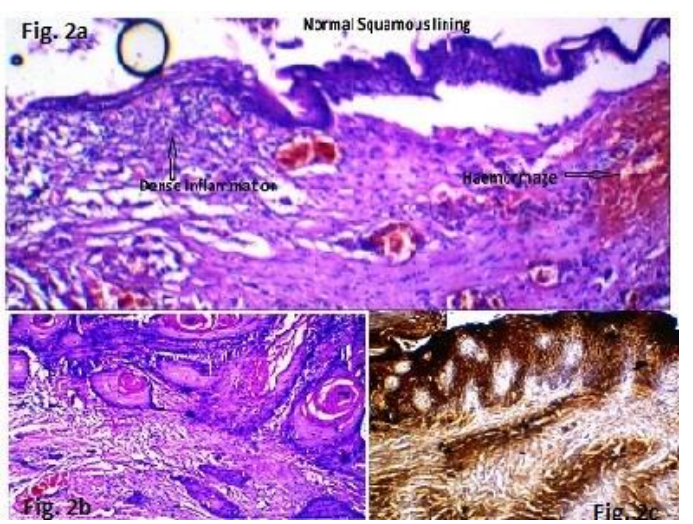

Fig. 2: Microphotograph showing (a) areas of normal squamous lining; (b) malignant squamous Component; and (c) malignant squamous component positive for Cytokeratin (40X)

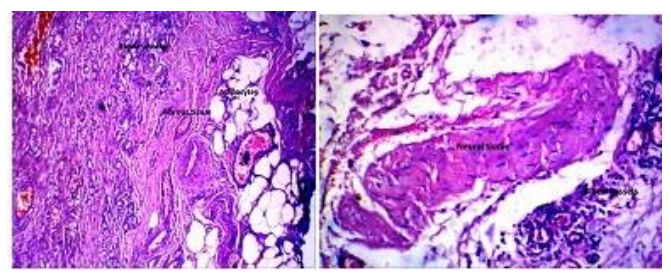

Fig. 3: Microphotograph showing other components of teratoms - adipocytes, blood vessels, fibrous tissue and neural tissue. (40 X)

\section{Discussion}

Germ cell tumours (GCTs) that arise outside the testes or the ovaries are classified as extragonadal. In children, sacrococcygeal teratomas comprise of $40 \%$ of all GCTs and up to $78 \%$ of all extragonadal GCTs. Sacrococcygeal teratoma is the most common solid neoplasm in neonates, with an estimated prevalence of 1 in 35000-40 000 live births with female to male ratio $4: 1{ }^{1}$ Adult sacrococcygeal teratoma is extremely rare with an incidence of $1: 87,000$ and a female to male ratio of $10: 1$. Most common are benign, also called mature teratomas. Sacrococcygeal teratomas with malignant elements generally are not seen in infants. Malignancy is very rare in adult sacrococcygeal teratomas and if present arises from the embryonic tissue. In the present case malignant change was noted in the squamous epithelium. Squamous cell carcinoma has been reported in only a single case. ${ }^{3}$ A total of 92 cases sacrococcygeal teratomas in adults, with only 20 as malignant or with malignant transformation are available in the literature. ${ }^{1,3-5}$ Most adult sacrococcygeal teratomas are cystic and benign and only $1-2 \%$ are malignant. The risk of malignancy increases with age. ${ }^{6}$ Mature Sacrococcygeal teratomas can undergo dysplastic change and frank malignant degeneration.

Sacrococcygeal teratomas are thought to originate from multipotent cells in Henson's node, which migrates caudally to rest in the coccyx. All Sacrococcygeal teratomas involve the coccyx. They may grow postero-inferiorly into the gluteal area and/or antero-superiorly into the abdomino-pelvic cavity. ${ }^{1,7}$ Most adult Sacrococcygeal teratomas are intra pelvic. ${ }^{4}$ However, there was no intra pelvic extension in the present case.

Teratomas often are comprised of cells that represent all three germ cell layers. They have solid, cystic or mixed components. Unlike teratomas in other locations, sacrococcygeal teratomas often do not have a capsule or pseudocapsule, which contributes to the difficulty in achieving a complete resection. Exclusively pre-sacral tumours present later than those with an external component, and have a higher prevalence of malignant transformation Solid tumours are more likely to be malignant. Necrosis, poor line of demarcation of the adjacent soft tissue planes, invasion of adjacent structures, destruction of sacrum, regional lymphadenopathy and distant metastases are signs suggestive of malignancy. ${ }^{1,4}$

Malignant teratomas, in addition to mature and/or embryonic tissues, have frankly malignant tissue of germ cell origin, such as germinoma and choriocarcinoma. Tumours containing malignant, non-germ cell elements, including 
adenocarcinoma and squamous cell carcinoma, are referred to as teratoma with malignant transformation. Patients with either a malignant teratoma or a benign teratoma with malignant transformation have a considerable increase in mortality. Patients with Sacrococcygeal teratomas may be asymptomatic on initial presentation or may manifest a variety of symptoms that are not indicative of either benign or malignant nature.

Imaging features alone do not allow definite differentiation between benign and teratomas with malignant transformation. Tumour markers, such as alpha-fetoprotein, carcino-embryonic antigen and human chorionic gonadotropin, are not helpful in differentiating between benign and malignant lesions. ${ }^{6}$

Surgical treatment of sacrococcygeal teratomas is complete excision, inclusive of coccyx and may be sacrum if involved. Coccyx may contain a nidus of pleuripotent cells that increases the recurrence rates to $37 \%$ when not excised. ${ }^{1,2,6}$ Most recurrences may be due to retained tissue, spillage of cyst contents during dissection or due to residual tissue densely adherent to the vital structures left behind at initial operation. Malignant tumours have a greater likelihood of recurrence and the incidence of malignancy is higher in recurrent than in primary tumours. ${ }^{[6]}$ If complete resection is accomplished, benign teratomas have a good prognosis. Since, malignant transformation is rare there has been no standard recommendation for the use of chemotherapy or radiotherapy and the best treatment plan is individualised multimodality therapy. ${ }^{1,8}$

Complete resection was not possible in this case and part of the tumour wall adherent with the sacrum was fulgurated with an advice for radiotherapy on follow-up.

\section{Conclusion}

Although a rare tumour in adults, sacrococcygeal teratoma should be considered in the differential diagnosis of patients with occult or a palpable pelvic mass.

\section{References}

1. Monteiro M, Cunha TM, Catarino A, Tome V. Sacrococcygeal teratoma with malignant transformation in an adult female: CT and MRI findings. Br J Radiol 2002;75:620-23.

2. Ng EW, Porcu P, Loehrer P. Sacrococcygeal teratoma in adults: case reports and a review of the literature. Cancer 1999;86[7]:1198-202.
3. Chen KT, Squamous cell carcinoma arising in sacrococcygeal teratoma. Arch Pathol Lab Med 1980;104 (6):336. PMID: 6892874

4. B. Kostas, G. Zisis, T. Christos, K. Isaak, G. Vrakas, X. Vrakas: Sacrococcygeal teratoma in adults: Report Of A Case And Literature Review. The Internet Journal of Surgery. 2007;11(2). DOI: 10.5580/fe3

5. Robin NH, Grace K, DeSousa TG, McDonaldMcGinn D, Zackai EH. New finding of SchinzelGiedion syndrome: a case with malignant sacrococcygeal teratoma. Am J Med Genet 1993;47:852-6.

6. Al-Essa AA, Malik TA, Baghdadi MK, El Tayeb AA. Adult sacrococcygeal teratomas. Saudi Med J. 2004 25(3):367-9. PMID: 15048179

7. Audet IM, Goldhahn RT Jr, Dent TL. Adult sacrococcygeal teratomas. Am Surg 2000;66.615.

8. Lopes A, Pearson S, Roberts J, et al. Case report: immature presacral teratoma in an adult female. Gynecol Oncol 1990;38:135-7. 